\title{
Neuromyopathy with congenital cataracts and glaucoma: a distinct syndrome caused by POLG variants
}

\author{
Claudia Castiglioni ${ }^{1}$ - Fabiana Fattori ${ }^{2}$ Bjarne Udd ${ }^{3,4,5}$ - Maria de los Angeles Avaria ${ }^{6}$ Bernardita Suarez ${ }^{1}$. \\ Adele $D^{\prime}$ Amico $^{2} \cdot$ Alessandro Malandrini ${ }^{7} \cdot$ Rosalba Carrozzo $^{2} \cdot$ Daniela Verrigni $^{2} \cdot$ Enrico Bertini $\mathbb{D}^{2}$. \\ Giorgio Tasca ${ }^{8}$
}

Received: 22 April 2017 / Accepted: 23 August 2017 / Published online: 22 January 2018

(c) European Society of Human Genetics 2018

\begin{abstract}
We identified three non-related patients manifesting a childhood-onset progressive neuromyopathy with congenital cataracts, delayed walking, distal weakness and wasting, glaucoma and swallowing difficulties. Electrophysiology and nerve biopsies showed a mixed axonal and demyelinating neuropathy, while muscle biopsy disclosed both neurogenic and myopathic changes with ragged red fibers, and muscle MRI showed consistent features across patients, with a peculiar concentric distoproximal gradient of fatty replacement. We used targeted next generation sequencing and candidate gene approach to study these families. Compound biallelic heterozygous variants, p.[(Pro648Arg)]; [(His932Tyr)] and p.[(Thr251Ile),(Pro587Leu)]; [(Arg943Cys)], were found in the three patients causing this homogeneous phenotype. Our report on a subset of unrelated patients, that showed a distinct autosomal recessive childhood-onset neuromyopathy with congenital cataracts and glaucoma, expands the clinical spectrum of POLG-related disorders. It also confirms the association between cataracts and neuropathy with variants in POLG. Early onset cataract is otherwise rare in POLG-related disorders and so far reported only in a few patients with the clinical pattern of distal myopathy or neuromyopathy.
\end{abstract}

Claudia Castiglioni and Fabiana Fattori contributed equally to this work.

Electronic supplementary material The online version of this article (https://doi.org/10.1038/s41431-017-0003-4) contains supplementary material, which is available to authorized users.

\section{Enrico Bertini}

ebertini@tin.it

1 Department of Pediatric Neurology, Clinica Las Condes, Santiago, Chile

2 Unit of Neuromuscular and Neurodegenerative Disorders, Bambino Gesù Children's Research Hospital, Rome, Italy

3 University of Helsinki, Folkhälsan Institute of Genetics, Helsinki, Finland

4 Neuromuscular Research Center, Tampere University and University Hospital, Tampere, Finland

5 Department of Neurology, Vasa Central Hospital, Vaasa, Finland

6 University of Chile Pediatric Neurology Santiago de Chile, Santiago de Chile, Chile

7 Department of Medicine, Surgery and Neuroscience, University of Siena, Siena, Italy

8 Istituto di Neurologia, Università Cattolica del Sacro Cuore, Fondazione Policlinico Universitario “A. Gemelli”, Rome, Italy

\section{Introduction}

Mitochondrial disorders are among the most frequent inherited metabolic diseases. Despite this, they are not always readily recognized and correctly managed, being extremely heterogeneous, both clinically and genetically. Variants in POLG, which is the gene coding for DNA polymerase gamma, an enzyme involved in the replication of human mitochondrial DNA, are known to cause a wide array of pathologies, ranging from progressive external ophthalmoplegia (PEO) to fatal Alpers syndrome. More than 200 disease causing variants in $P O L G$ inherited as autosomal dominant or recessive traits have been published [1]. Variants that affect function are scattered throughout the coding region of $P O L G$, and genotype-phenotype correlation is not always predictable [2], with some exceptions constituted by some common variants in the linker region, such as the c.1399 G>A (p.(Ala467Thr)) which is known to occur more frequently in the childhood-onset cases with Alpers syndrome [3], the c.2243 G > C (p.(Trp748Ser)) related to recessive ataxia, and the c.1760 C $>\mathrm{T}$ (p. (Pro587Leu)), that is often found on the same allele with the c.752 C > T (p.(Thr251Ile)) [4] and whose main clinical 


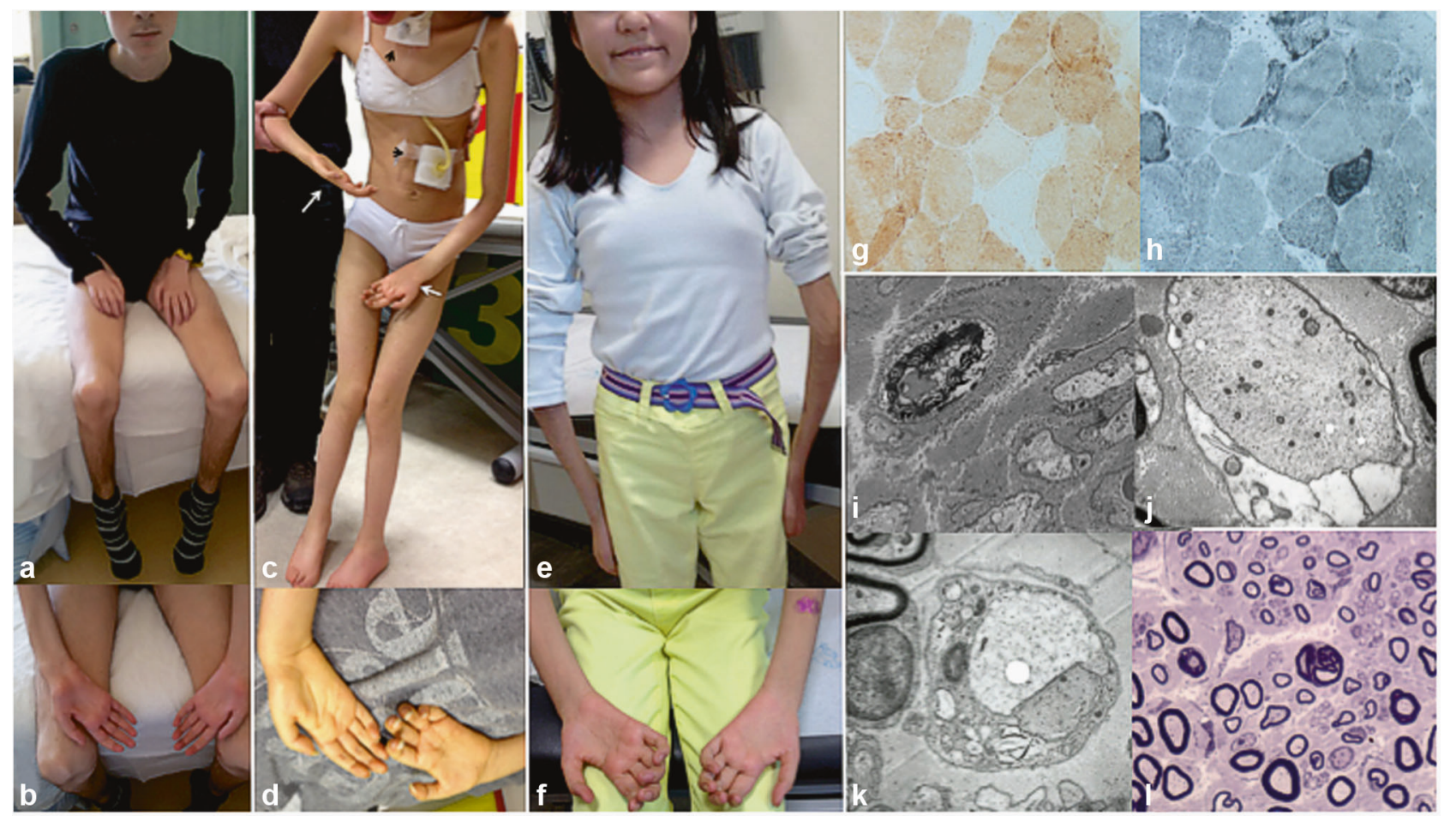

Fig. 1 Clinical and histopathological features. a-f Clinical manifestations of patient $1(\mathbf{a}, \mathbf{b})$ patient $2(\mathbf{c}, \mathbf{d})$ and patient $3(\mathbf{e}, \mathbf{f})$ showing similar aspects of severe distal amyotrophy and paralysis of hands and feet; note gastrostomy in patient 2 (d). Figure $\mathbf{g}$ and $\mathbf{h}$ correspond to muscle pathology of patient 1 , with seriate sections stained for cytochrome c oxidase (COX) (g) and succinate dehydrogenase (SDH) (h) showing COX negative fibers with marked mitochondrial proliferation on SDH staining. Figures $\mathbf{i}-\mathbf{l}$ illustrate nerve pathology, with electron microscopy images of patient 2 (i) obtained late in the disease course, and of patient $1(\mathbf{j}, \mathbf{k})$ and light microscopy of semithin sections of patient 1 obtained at early stages of the disease at age 9 years. (l) Figure 1i shows a single myelinated axon with relative thin myelin; this was a single myelinated axon found in the nerve biopsy of patient 2 at the age of 25 years, that besides showed a marked subtotal loss of myelinated axons. Figure $1 \mathrm{j}$ shows a demyelinated fiber, and Fig. 1k shows a demyelinated fiber with aspects of degeneration and myelinophagia. Figure 11 shows reduced number of myelinated axons, some of which show a thinner myelin in relation to the axonal diameter picture is represented by recessive PEO [5, 6]. Likewise, the majority of the variants causing dominant PEO seem to cluster in the polymerase domain [5].

Lately, a new $P O L G$-related phenotype characterized by slowly progressive mitochondrial distal myopathy and cataracts was described. This seems to be a relatively rare entity reported in single cases with dominant inheritance associated with cachexia [7-9]. Early onset cataract appears to be very rare in $P O L G$-mutated patients and has been so far reported either in a few patients with recessive $P O L G$ variants $[5,6]$ or, noteworthy, in 3 of the above-mentioned distal myopathy patients harboring dominant missense variants in exon 17 , c. $2687 \mathrm{~T}>\mathrm{G}$ (p. (Leu896Arg)) or involving a different amino acid change in the same position (either p.(Tyr951Asn) or p.(Tyr951His)) $[8,9]$.

Here, we describe a distinct recessive childhood-onset phenotype in three unrelated sporadic patients, partially overlapping with the dominant mitochondrial distal myopathy with cachexia, and characterized by sensory-motor neuropathy, mitochondrial myopathy, congenital cataracts, glaucoma and homogeneous findings on muscle imaging.
We also provide a summary of other published patients with distal myopathy or neuromyopathy with cataracts and variants in $P O L G$.

\section{Patients and methods}

\section{Patients}

Patient 1 is followed at the Bambino Gesù Children's Research Hospital, while patients 2 and 3 are followed at Clinica Las Condes, Chile. The patients' families gave their consent for publication of clinical information and pictures. Informed consent from parents was obtained before the molecular genetic diagnostic work-up. The Ethics Committee of the involved institutions approved the research protocol.

\section{Muscle and nerve biopsies}

Frozen muscle sections from patient 1 were stained according to standard procedures [10]. Analysis by electron 
Fig. 2 Muscle MRI. T1weighted MRI images of thighs and lower legs in patients 1 (a) and 2 (b). Note the fatty replacement that progresses from outer to inner muscle layers particularly evident in the anterior thigh compartments (arrows). In this context, there is relative sparing of deeper muscles such as adductor magnus (asterisk) and tibialis posterior (arrowheads)

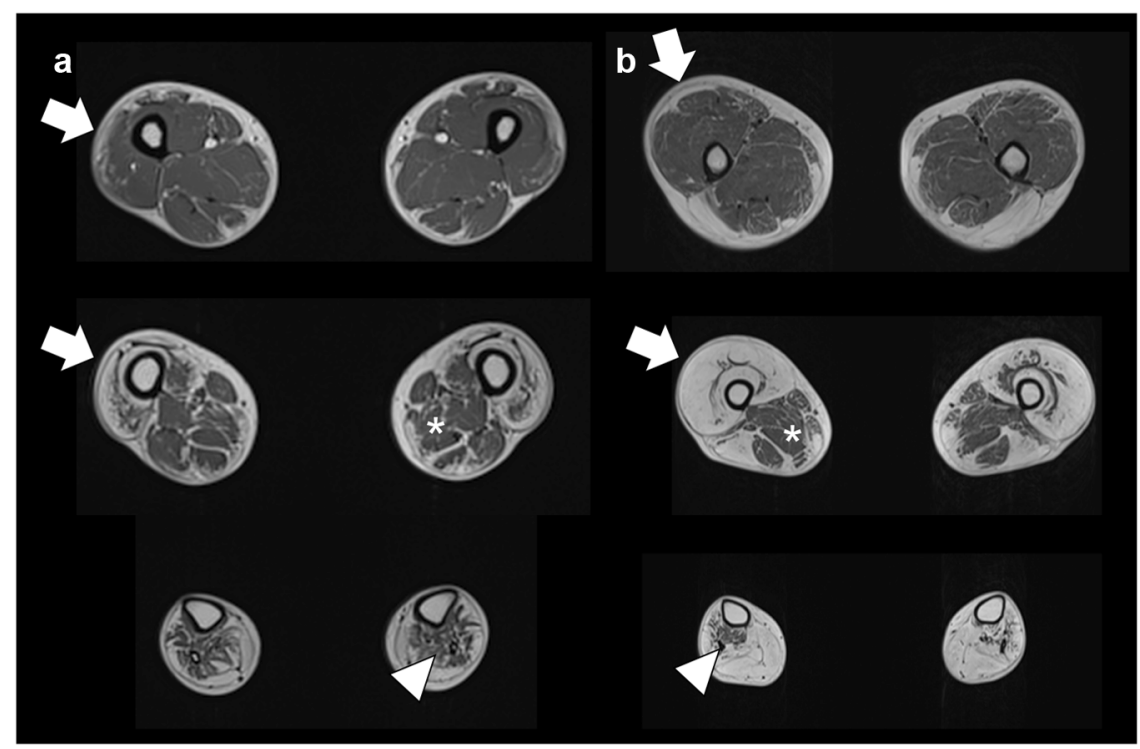

microscopy according to standard protocols was used for better characterization of ultrastructural abnormalities of the performed nerve biopsies.

To detect multiple deletions, long-PCR analysis in DNA extracted from muscle biopsy of index patient 1 , and, for comparison, from another patient positive for single deletion was performed. Forward primer-3.701 and backward primer-16.042 were used.

\section{Muscle magnetic resonance imaging (MRI)}

Muscle MRI of the pelvis and lower limbs was performed using conventional T1-weighted and STIR sequences on $1.5 \mathrm{~T}$ scanners according to a protocol previously reported [11].

\section{Molecular genetics}

Genomic DNA was extracted from peripheral blood in all patients using DNA Extraction Kit (Qiagen, Hilden, Germany). A targeted next generation sequencing was performed in patients 1 and 2 with an identical technical setup of MyoCap but including all known genes for neuropathies and motor neuron disorders [12].

Sanger sequencing was performed to confirm results obtained by NGS analysis in patients 1 and 2 and to investigate family members of the probands for the presence of the variants identified. The genomic sequence of $P O L G$ was used to design PCR primer pairs to amplify all 22 coding exons and flanking intronic regions, as previously described [6]. Nucleotide sequence determination was performed by cycle sequencing using a BigDye Terminator DNA sequencing kit v3.1 (Applied Biosystems, Hercules, CA, USA). DNA sequences were analyzed using Seqscape software version 3.0 and the GenBank POLG sequence (ID NM_002693.1).

All POLG variants reported in this study were entered into the Leiden Open Variation Database (www.LOVD.nl/ POLG; patient IDs 00105907, 00105904, 00105903).

\section{Results}

\section{Case presentations}

\section{Patient 1}

This boy was born from non-consanguineous Southern Italian parents, and underwent bilateral cataract surgery at age 6 months. He manifested delayed walking ability and progressive distal upper and lower limbs weakness. Dysphagia followed by strabismus and ptosis appeared at 12 and 15 years, respectively. At age 16, he presented an acute glaucoma in the left eye, and since then he has been pharmacologically treated. When last examined at age 20, he was able to walk only with ankle-foot orthoses. He had facial weakness, tongue hypotrophy, ptosis and strabismus without ophthalmoparesis. Proximal upper and lower limb strength was slightly reduced while distal muscles including intrinsic hand ones were severely weak (Fig. 1a, b). Deep tendon reflexes were absent, and sensation was normal. A sural nerve biopsy performed at the age of 9 showed, on semithin sections and electron microscopy, reduction of myelinated axons, figures of myelin outfoldings and some axons showing a thin myelin sheath for axonal diameter (Fig. 1j-1). Electrophysiological examination at age 12 years showed a sensorimotor neuropathy with reduced motor and sensory conduction velocities: sensory action 
potential of the sural nerve was absent, peroneal nerve conduction velocity was $10 \mathrm{~m} / \mathrm{s}$ and compound muscle action potential (CMAP) amplitude of extensor digitorum brevis muscle was reduced at $0.3 \mathrm{mV}$. Brain MRI was unremarkable.

Muscle MRI showed a pattern of fatty replacement that was not selective for specific muscles but rather suggestive of a distal-proximal and "concentric" gradient of fatty replacement, with the outer part of each muscle more involved than the inner part (Fig. 2).

Muscle biopsy disclosed the presence of both chronic neurogenic and myopathic changes with ragged red fibers (RRF) and multiple deletions of mitochondrial DNA (Fig. $1 \mathrm{~g}$, h, and Supplementary Information, Fig. 1).

\section{Patient 2}

This Chilean girl was born healthy at term from nonconsanguineous parents and underwent surgery for bilateral cataracts at age 3 months. By 8 months of age she developed motor delay and walked with orthoses at age 4 years. Electrophysiological examination performed at age 3 years disclosed significant slowing of motor velocities in the range of $25-35 \mathrm{~m} / \mathrm{s}$ in median and ulnar nerves and $41 \mathrm{~m} / \mathrm{s}$ in peroneal nerves. The motor responses showed extremely reduced amplitude of $0.2 \mathrm{mV}$ in extensor digitorum brevis and thenar muscles with normal sensory conductions of sural and ulnar nerves. She underwent gastrostomy for swallowing difficulties, and tracheostomy for respiratory failure with recurrent infections at the age of 20 and 23 years, respectively. When last examined at age 25 , she had severe generalized muscle wasting with distal tetraparesis, tongue atrophy, thick lips, marked bilateral exophthalmos, ophthalmoparesis and bilateral blindness caused by acute glaucoma at age 15 years (Fig. 1c, d). Cerebrospinal fluid (CSF) analysis showed hyperproteinorrachia $3.15 \mathrm{~g} / 1$ (normal 0.15-0.6) and the muscle biopsy performed at age 12 years described nonspecific myopathic changes but SDH/ COX staining was not performed. Brain MRI only detected very mild cerebellar vermis atrophy. A nerve biopsy at age 25 years showed prominent axonal loss with secondary myelin abnormalities in very few residual axons on electron microscopy (Fig. 1i).

\section{Patient 3}

This Chilean girl was born at term from nonconsanguineous parents. She was unrelated with the previous family, coming from a different geographical area. She was evaluated elsewhere for the first time at 24 months of age by a pediatric neurologist since developmental delay and hypotonia was manifest. Only at this evaluation bilateral cataracts were detected and addressed to ophthalmologist. At that time a muscle biopsy described myopathic changes with RRF. CSF analysis disclosed normal lactate and elevated protein levels $6.5 \mathrm{~g} / \mathrm{l}$, which led to the diagnosis of chronic inflammatory demyelinating neuropathy. She was treated with intravenous immunoglobulins without clinical improvement. Nerve conduction velocity studies performed at age 9 were consistent with a sensory-motor neuropathy. Absent sural nerve action potentials, prolonged distal latency on median and peroneal nerves, reduced CMAP amplitude $(0.1-0.2 \mathrm{mV}$ in extensor digitorum brevis and thenar muscles, respectively), and slowing of conduction velocities $(9-15 \mathrm{~m} / \mathrm{s})$ were detected. At age 12 years, she underwent corneal implant on the right eye following an acute glaucoma with corneal damage. She was investigated for lack of development of secondary sexual characters with a diagnosis of functional hypogonadotropic hypogonadism. When re-evaluated at 16 years her hands were paretic and severely atrophied, adopting a fixed supine posture with the palms of the hands facing permanently upwards without proximal radioulnar joint movements (Fig. 1e, f). Mild ptosis, ophthalmoparesis, and facial weakness were also present. Muscle MRI features were very similar to patient 1 (Fig. 2). Brain MRI was normal.

None of our patients showed any clinical sign or laboratory finding suggestive of ataxia, epilepsy, or hepatopathy.

\section{Molecular genetic analyses}

Using a candidate gene approach in patient 1, based on clinical and electrophysiological findings, variants in DNM2 and MFN2 were first ruled out by direct sequencing. Targeted next generation sequencing then detected two missense variants in $P O L G$ : c. $1943 \mathrm{C}>\mathrm{G}$ (p.(Pro648Arg)) in exon 10 and c.2794 $\mathrm{C}>\mathrm{T}$ (p.(His932Tyr)) in exon 18 (exons are numbered like in reference [6) that were confirmed by Sanger sequencing and segregated on two different alleles in the parents. In patient 2 direct sequencing ruled out variants in $D N M 2$, responsible of a form of neuropathy and cataracts [13], in CTDP1 [14], and MTMR2, known to cause the Charcot-Marie Tooth type 4B variant with thick lips [15]. Later, the same next generation sequencing approach identified the c.2827 C $>\mathrm{T}$ (p. (Arg943Cys)) variant in exon 18. Given the similarity of the phenotype with patient 1 , Sanger sequencing was performed to complete the POLG sequence that was not entirely covered by NGS approach and two other variants, c. $1760 \mathrm{C}$ $>\mathrm{T}$ (p.(Pro587Leu)) in exon 10 and c.752 C > T (p. (Thr251Ile)) in exon 3, were found on the other allele, segregating appropriately in parents.

Patient 3 showed very similar clinical features so $P O L G$ was directly sequenced finding the same compound 


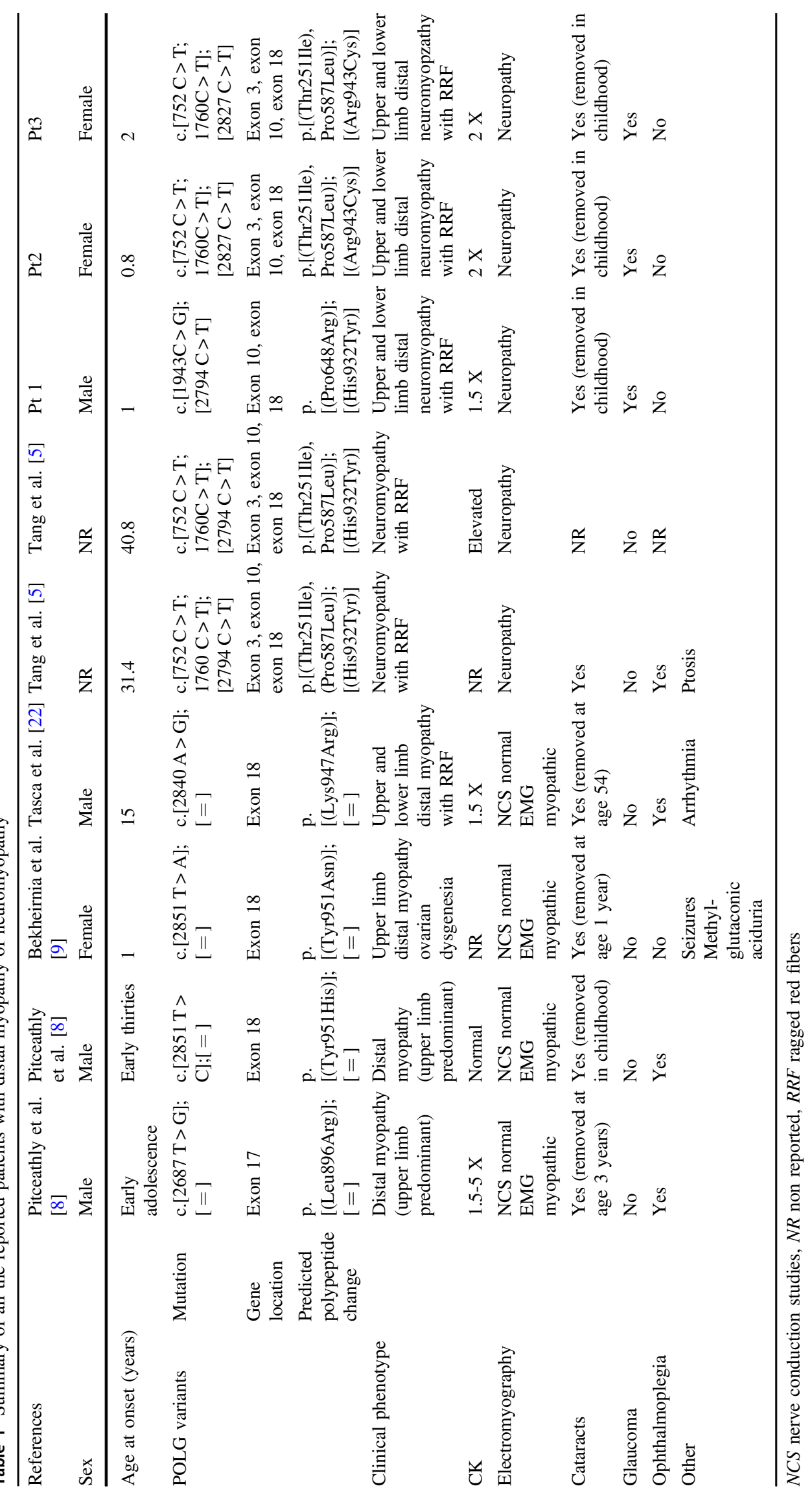


heterozygous variants of patient 2, c.1760 C>T (p. (Pro587Leu)), in cis with the c.752 C > T (p.(Thr251Ile)), and c. $2827 \mathrm{C}>\mathrm{T}$ (p.(Arg943Cys)) (Supplementary Information, Fig. 2). The patients' parents were heterozygous carriers of the identified variants.

Table 1 summarizes the features of our patients and of other patients reported as affected by a distal myopathy or neuromyopathy, associated with early onset cataracts and variants in $P O L G$.

\section{Discussion}

We identified three patients with compound heterozygous variants in POLG causing a homogeneous phenotype of severe neuromyopathy with cataracts and glaucoma.

These patients harbor a combination of variants in $P O L G$ exons 18 and 10. The c. $1760 \mathrm{C}>\mathrm{T}$ (p.(Pro587Leu)) is associated in two patients with another variant in exon 3 on the same allele: c.752 C> T (p.(Thr251Ile)). This allele is reported among the most frequently and widely described POLG variants $[3,6,16,17,18]$, and the two substitutions are thought to act synergistically to affect POLG function [18].

In all three patients the onset was characterized by very early cataracts, delayed walking and distal weakness. Later in the second decade all patients developed glaucoma. Muscle biopsy showed myopathic or mixed myopathic and neurogenic findings with RRF, and muscle MRI showed consistent features across patients. The polyneuropathy in these patients is characterized by a sensory-motor, predominantly axonal with associated demyelinating involvement, associated with a severe distal muscle atrophy progressing into paralysis of hands and feet. Electrophysiological examinations showed that motor and sensory conduction velocities were markedly slowed with reduced CMAP amplitude in distal muscles of the legs. This polyneuropathy was concomitant with a primary mitochondrial myopathy characterized by slightly elevated CK levels, RRF, and multiple mitochondrial DNA deletions on muscle biopsy. Cachexia was also a typical feature, probably due to dysphagia as well as to the progressive neuromyopathy. Hyperproteinorrachia in CSF was also a feature, and hypogonadism, that was present in one of our patients, has been reported in association with early onset cataracts in a single patient with a heterozygous variant in POLG [9]. No additional manifestations reported in POLG-related disorders were observed: patients had a normal intelligence, with a substantially normal brain MRI, without epilepsy or heart involvement.

Congenital cataract represents a rare feature reported in other neurological/neuromuscular disorders such as Marinesco-Sjogren syndrome and congenital cataracts and facial dysmorphism (CCFDN) but rarely associated with POLG variants [5, 6, 8, 9]. Interestingly, the c. $2827 \mathrm{C}>\mathrm{T}$ (p.(Arg943Cys)) variant in the Chilean patients of our cohort, already associated with PEO and parkinsonism combined with a variant in the exonuclease domain of POLG [19], has been also described in a young girl with developmental delay, dysmorphic features, and congenital cataract in association with a variant in the linker region domain [6]. Early onset cataract has been also reported in another patient with primary ovarian failure and PEO harboring a dominant variant (c.2828 $\mathrm{G}>\mathrm{A}$ ) affecting the same Arg943 residue (p.(Arg943His)) [16]. This finding might suggest a genotype-phenotype correlation between this clinical feature and this arginine residue in the polymerase domain, which is known to be involved in binding the oxygen atoms of the gamma-phosphate of the incoming dNTP and in helping to position the incoming dNTP for catalysis [6].

The association of glaucoma and cataracts is unique and has not been so far related to POLG. A possible link between mitochondrial dysfunction and pathogenesis of glaucoma-induced blindness is provided by recent evidences suggesting that retinal ganglion cells, that are particularly dependent on mitochondrial metabolism, are also highly susceptible to damage in open-angle glaucoma. Oxidative injury inducing vascular alterations of the optic nerve or causing dysfunctions of the trabecular meshwork with consequent aqueous humor outflow impairment are additional factors contributing to the pathogenesis of glaucoma [20, 21].

The differential diagnosis of this distinct syndrome includes the neuropathy with CCFDN associated with a founder variant in CTDPl in the gypsies [14]. In this disease, however, the neuropathy is purely demyelinating and glaucoma is absent. Similarly, variants in DNM2 can cause neuropathy, myopathy, and cataracts without glaucoma [13]. Variants in two myotubularin-related genes, namely MTMR2 and MTMR13, cause demyelinating neuropathy with myelin outfoldings, facial, and bulbar weakness (CMT4B1) or with glaucoma but no cataracts (CMT4B2) [15]. In our patients, concomitant variants in these genes were excluded either by direct sequencing or because these genes were included in the next generation sequencing panel.

The phenotype we described also shares some features with the reported $P O L G$-distal myopathy with cataracts (Table 1). In our literature review of this condition, we found that three patients out of four showed onset of cataracts in childhood, while one had later onset, and none of them had a neuropathy. Heterozygous dominant variants were detected in all unrelated individuals, all were located in exons 17 or 18 of the polymerase domain, and two of these variants changed the same Tyr951 residue with a 
different amino acid (Histidine and Asparagine). Interestingly, all of our three patients also showed one heterocompound variant in exon 18. Tang and collaborators [5] also described two patients harboring the same exon 18 variant of patient 1 , c.2794 C > T (p.(His932Tyr)), in combination with the same in cis variants of patients 2 and $3 \mathrm{c}$. [1760 C> T; $752 \mathrm{C}>\mathrm{T}$ ] (p.(Pro587Leu); (Thr251Ile)). Interestingly, these patients share several clinical features with ours, such as neuromyopathy, and cataracts in one case but not glaucoma (Table 1).

Of note, muscle imaging was similar in two of our patients, consistently displaying the concentric and distoproximal gradient, which is reminiscent of the pattern described in distal myopathy patients with dominant $P O L G$ variants [8, 22]. This MRI pattern, which is unique and not reported in any other neuromuscular disorder, could be considered as a recognizable biomarker to prioritize a genetic screening of $P O L G$ in undiagnosed patients with neuropathy or distal myopathy.

In conclusion, this report expands the clinical spectrum of POLG-related disorders, and identifies a distinct phenotype of severe neuromyopathy with congenital cataracts and glaucoma. Intraocular pressure should be carefully measured in patients with POLG variants because all of them may be at risk of developing overt glaucoma, and antioxidant drugs might have a protective effect on glaucomainduced optic nerve damage in these patients.

Acknowledgements We thank the patients' families for their participation to the study. This work was supported by a grant from Ministry of Health, Ricerca Corrente to $\mathrm{EB}, \mathrm{FF}$, and $\mathrm{AD}$, and a grant from Clinica Las Condes PIDA-2014-002 to CC. We are indebted to the technician Margherita Verardo who contributed to the histopathological processing of muscle biopsies.

\section{Compliance with ethical standards}

Conflict of interest The authors declare that they have no competing interests.

\section{References}

1. Cohen BH, Chinnery PF, Copeland WC. POLG-related disorders. 16 Mar 2010 [updated 18 Dec 2014]. In: Pagon RA, Adam MP, Ardinger HH, Wallace SE, Amemiya A, Bean LJH, Bird TD, Fong CT, Mefford HC, Smith RJH, Stephens K, editors. GeneReviews $^{\circledR}$ [Internet]. Seattle (WA). Seattle: University of Washington; 1993-2016. Available from http://www.ncbi.nlm. nih.gov/books/NBK26471/.

2. Milone M, Massie R. Polymerase gamma 1 mutations: clinical correlations. Neurologist. 2010;16:84-91.

3. Horvath R, Hudson G, Ferrari G, et al. Phenotypic spectrum associated with mutations of the mitochondrial polymerase gamma gene. Brain. 2006;129:1674-84.

4. DeBalsi KL, Longley MJ, Hoff KE, Copeland WC. Synergistic effects of the in cis T251I and P587L mitochondrial DNA polymerase $\gamma$ disease mutations. J Biol Chem. 2017;292:4198-4209.

5. Tang S, Wang J, Lee NC, et al. Mitochondrial DNA polymerase gamma mutations: an ever expanding molecular and clinical spectrum. J Med Genet. 2011;48:669-81.

6. Wong LJ, Naviaux RK, Brunetti-Pierri N, et al. Molecular and clinical genetics of mitochondrial diseases due to POLG mutations. Hum Mutat. 2008;29:E150-E172.

7. Giordano C, Pichiorri F, Blakely EL, et al. Isolated distal myopathy of the upper limbs associated with mitochondrial DNA depletion and polymerase gamma mutations. Arch Neurol. 2010;67:1144-46.

8. Pitceathly RD, Tomlinson SE, Hargreaves I, et al. Distal myopathy with cachexia: an unrecognised phenotype caused by dominantly-inherited mitochondrial polymerase $\gamma$ mutations. $\mathbf{J}$ Neurol Neurosurg Psychiatry. 2013;84:107-10.

9. Bekheirnia MR, Zhang W, Eble T, et al. POLG mutation in a patient with cataracts, early-onset distal muscle weakness and atrophy, ovarian dysgenesis and 3-methylglutaconic aciduria. Gene. 2012;499:209-12.

10. Tasca G, Ricci E, Penttilä S, et al. New phenotype and pathology features in MYH7-related distal myopathy. Neuromuscul Disord. 2012;22:640-47.

11. Tasca G, Ricci E, Monforte M, et al. Muscle imaging findings in GNE myopathy. J Neurol. 2012;259:1358-65.

12. Evilä A, Arumilli M, Udd B, Hackman P. Targeted nextgeneration sequencing assay for detection of mutations in primary myopathies. Neuromuscul Disord. 2016;26:7-215.

13. Jungbluth H, Cullup T, Lillis S, et al. Centronuclear myopathy with cataracts due to a novel dynamin 2 (DNM2) mutation. Neuromuscul Disord. 2010;20:49-52.

14. Lassuthova P, Sišková D, Haberlová J, Sakmaryová I, Filouš A, Seeman P. Congenital cataract, facial dysmorphism and demyelinating neuropathy (CCFDN) in 10 Czech Gypsy children--frequent and underestimated cause of disability among Czech Gypsies. Orphanet J Rare Dis. 2014;1(9):46.

15. Tazir M, Bellatache M, Nouioua S, Vallat JM. Autosomal recessive Charcot-Marie-Tooth disease: from genes to phenotypes. J Peripher Nerv Syst. 2013;18:113-29.

16. Blok MJ, van den Bosch BJ, Jongen E, Hendrickx A, de DieSmulders CE, Hoogendijk JE, Brusse E, de Visser M, Poll-The BT, Bierau J, de Coo IF, Smeets HJ. The unfolding clinical spectrum of POLG mutations. J Med Genet. 2009;46:776-85.

17. Mancuso M, Filosto M, Oh SJ, DiMauro S. A novel polymerase gamma mutation in a family with ophthalmoplegia, neuropathy, and Parkinsonism. Arch Neurol. 2004;61:1777-79.

18. Ferreira M, Evangelista T, Almeida LS, et al. Relative frequency of known causes of multiple mtDNA deletions: two novel POLG mutations. Neuromuscul Disord. 2011;21:483-88.

19. Sato K, Yabe I, Yaguchi H, et al. Genetic analysis of two Japanese families with progressive external ophthalmoplegia and parkinsonism. J Neurol. 2011;258:1327-32.

20. Osborne NN, Núñez-Álvarez C, Del Olmo-Aguado S, MerrayoLloves J. Visual light effects on mitochondria: The potential implications in relation to glaucoma. Mitochondrion. 2016;S15677249(16):30258-6.

21. Pinazo-Durán MD, Zanón-Moreno V, Gallego-Pinazo R, GarcíaMedina JJ. Oxidative stress and mitochondrial failure in the pathogenesis of glaucoma neurodegeneration. Prog Brain Res. 2015;220:127-53.

22. Tasca G, Monforte M, Nesti C, Santorelli FM, Silvestri G, Ricci E. Concentric muscle involvement in POLG-related distal myopathy. Neuromuscul Disord. 2017;27:500-01. 